Pacific Journal of Mathematics

PROPERTIES WHICH NORMAL OPERATORS SHARE WITH 


\title{
PROPERTIES WHICH NORMAL OPERATORS SHARE WITH NORMAL DERIVATIONS AND RELATED OPERATORS
}

\author{
JoEl ANDERSON AND CIPRIAN FoIAȘ
}

Let $S$ and $T$ be (bounded) scalar operators on a Banach space $\mathscr{X}$ and let $C(T, S)$ be the map on $\mathscr{B}(\mathscr{X})$, the bounded linear operators on $\mathscr{X}$, defined by

$$
C(T, S)(X)=T X-X S
$$

for $X$ in $\mathscr{B}(\mathscr{X})$. This paper was motivated by the question: to what extent does $C(T, S)$ behave like a normal operator on Hilbert space? It will be shown that $C(T, S)$ does share many of the special properties enjoyed by normal operators. For example it will be shown that the range of $C(T, S)$ meets its null space at a positive angle and that $C(T, S)$ is Hermitian if $T$ and $S$ are Hermitian. However, if $\mathscr{X}$ is a Hilbert space then $C(T, S)$ is a spectral operator if and only if the spectrum of $T$ and the spectrum of $S$ are both finite.

We now indicate our results in greater detail. Let $\mathscr{H}$ be a Hilbert space and let $N$ be a normal operator in $\mathscr{B}(\mathscr{H})$. Then $N$ enjoys the following properties.

(A) $\mathscr{R}(N)$ is orthogonal to $\mathscr{N}(N)$, where $\mathscr{R}(N)(\mathscr{N}(N))$ donotes the range (null space) of $N$.

(B) $\mathscr{R}(N)^{-} \oplus \mathscr{N}(N)=\mathscr{H}$, where the bar denotes norm closure.

(C) There is a resolution of the identity $E(\cdot)$ supported by $\sigma(N)$ such that

$$
N=\int_{\sigma(N)} \lambda d E,
$$

where $\sigma(N)$ denotes the spectrum of $N$. That is, $N$ is a scalar operator.

(D) If $x \in E(\{\lambda\}) \mathscr{H}$ for some complex number $\lambda$, then $N x=\lambda x$.

(E) $N$ has closed range if and only if 0 is an isolated point in $\sigma(N)$. (We adopt the convention that 0 is isolated in $\sigma(N)$ if $0 \notin \sigma(N))$.

(F) The norm, spectral radius, and numerical radius of $N$ are equal.

(G) The closure of the numerical range of $N$ is the convex hull of the spectrum of $N$.

In $\S \S 1,2$, and 3 of this paper we show that appropriate versions of (A), (D), and (E) hold for $C(T, S)$. In Section 4 we restrict ourselves to the Hilbert space case and show that (B) is false in 
general. This result enables us to give the characterization of operators of the form $C(T, S)$ which are spectral operators which was mentioned above. In $\S 5$ assuming that $T$ and $S$ are normal operators on a Hilbert space, we show that $(G)$ holds but that in general (F) is false for $C(T, S)$. We conclude the paper with an example of a Hermitian operator whose square is not Hermitian.

In the sequel an operator shall be called spectral (scalar) if it is spectral (scalar) in the sense of Dunford. An operator shall be called Hermitian if it is Hermitian in the sense of Lumer and Vidav (see [7]). We shall make use of the theory of decomposable operators as presented in [3]. If $T$ is a decomposable operator on a Banach space $\mathscr{P}$ and $F$ is a closed subset of the complex plane $C$ (or the real line $\boldsymbol{R}$ ) we shall usually denote the spectral maximal subspace of $\mathscr{X}$ associated with $T$ and $F$ by $\mathscr{X}_{T}(F)$. However, the spectral maximal subspace of $\mathscr{B}(\mathscr{C})$ which is associated with $C(T, S)$ and the complex set $F$ shall be denoted by $\mathscr{B}_{C}(F)$. The derivation $C(T, T)$ shall sometimes also be written as $\Delta_{T}$. Following [1], if $N$ is a normal operator on a Hilbert space we shall call $\Delta_{N}$ the normal derivation induced by $N$.

1. It is shown in [1] that if $N$ is a normal operator in $\mathscr{B}(\mathscr{H})$ then

$$
\left\|Y-\Delta_{N}(X)\right\| \leqq\|Y\|
$$

for all $X$ in $\mathscr{B}(\mathscr{H})$ and all $Y$ in $\mathscr{N}\left(\Delta_{N}\right)$. In this section we obtain a generalized inequality for $C(T, S)$. Since the proofs are generally similar to those given in [1], we will be brief.

Definition 1.1. Let $\mathscr{C}$ and $\mathscr{N}$ be (not necessarily closed) subspaces of a normed linear space $\mathscr{Z}$. We shall say that $\mathscr{C}$ meets $\mathscr{N}$ at angle $\theta(0 \leqq \theta \leqq \pi / 2)$ where by definition

$$
\sin \theta=\inf \{\|m+n\|: m \in \mathscr{L}, n \in \mathscr{N},\|n\|=1\} .
$$

If $\mathscr{C}$ meets $\mathscr{N}$ at angle $\pi / 2$ we say that $\mathscr{M}$ is orthogonal to $\mathscr{N}$. It is easy to show that $\mathscr{C}$ meets $\mathscr{N}$ at angle 0 if and only if $\mathscr{N}$ meets $\mathscr{M}$ at angle 0 so that if $\mathscr{M}$ meets $\mathscr{N}$ at angle $\alpha>0$ then $\mathscr{N}$ meets $\mathscr{C}$ at angle $\beta>0$. In general, however, $\alpha$ need not equal $\beta$.

If $T$ is an invertible element of $\mathscr{B}(\mathscr{Z})$ then $T$ is said to be power bounded by $K$ if for some $K \geqq 1,\left\|T^{n}\right\| \leqq K$ for $n=$ $\pm 1, \pm 2, \cdots$.

Lemma 1.2. If $T$ and $S$ are invertible elements of $\mathscr{B}(\mathscr{X})$ 
which are both power bounded by $K$ then $\mathscr{R}(C(T, S))$ meets $\mathscr{N}(C(T, S))$ at angle $\theta$, where $\sin \theta \geqq 1 / K^{2}$.

Lemma 1.3. If $S$ and $T$ are in $\mathscr{B}(\mathscr{Q})$ and $i \notin \sigma(T) \cup \sigma(S)$ then $\mathscr{R}(C(T, S))=\mathscr{R}(C(W, V))$ where $W=(T-i I)(T+i I)^{-1}$ and $V=$ $(S-i I)(S+i I)^{-1}$ are the Cayley transforms of $T$ and $S$ respectively.

LEMma 1.4. Let $T$ and $S$ be scalar elements of $\mathscr{B}(\mathscr{Q})$ and let $f$ and $g$ be real-valued Borel measurable functions on $C$ which assume only finitely many (real) values. Then the Cayley transforms of $f(T)$ and $g(S)$ are both power bounded by $K$ for some $K>0$. Furthermore, the constant $K$ does not depend on the particular choice of $f$ or $g$.

The proof of (1.2) depends on the following generalization of the identity used to prove (1.4) in [1]: If $X$ and $Y$ are in $\mathscr{B}(\mathscr{P})$ and $T Y=Y S$ then for each integer $n$

$$
n T^{n-1} Y=T^{n} X-X S^{n}-\sum_{k=0}^{n-1} T^{n-k-1}(T X-X S-Y) S^{k} .
$$

The proof of (1.3) is an obvious modification of the proof of (1.5) in [1]. (1.4) follows easily from [5, Theorem 7, p. 330] (the constant $K$ depends only on the norms of the spectral projections associated with $T$ and $S$ ). Note that if $T$ and $S$ are normal operators on Hilbert space $K$ may be taken to be 1 in (1.4).

THEOREM 1.5. If $S$ and $T$ are scalar operators then there is a real number $\theta>0$ such that the range of $C(T, S)$ meets the null space of $C(T, S)$ at angle $\theta$. If $T$ and $S$ are normal operators on a complex Hilbert space then $\mathscr{R}(C(T, S))$ is orthogonal to $\mathscr{N}(C(T, S))$.

Proof. Let $E(\cdot)$ and $F(\cdot)$ be the spectral resolutions of identity associated with $T$ and $S$ respectively. Partition $\sigma(T) \cup \sigma(S)$ into rectangles $\delta_{1}, \delta_{2}, \cdots, \delta_{n}$ and let $\lambda_{i}$ be the midpoint of $\delta_{i}$ for $i=$ $1,2, \cdots, n$. Let $X$ and $Y$ be in $\mathscr{B}(\mathscr{Z})$ and suppose $Y$ is in $\mathscr{N}(C(S, T))$. Consider

$$
\left\|Y-\sum_{k=1}^{n} \lambda_{k} E\left(\delta_{k}\right) X-X \sum_{k=1}^{n} \lambda_{k} F\left(\delta_{k}\right)\right\| .
$$

To prove the theorem, it suffices to show that $(*) \geqq \sin \theta\|Y\|$ for some $\theta>0$ and all possible partitions of $\sigma(T) \cup \sigma(S)$. As in [1, (1.6)], a computation shows that the range of the map $X \mapsto$ $\sum_{k=1}^{n} \lambda_{k} E\left(\delta_{k}\right) X-X \sum_{k=1}^{n} \lambda_{k} F\left(\delta_{k}\right)$ does not change if the $\lambda_{k}$ 's aro 
replaced by any complex $n$-tuple $\left\{\mu_{1}, \mu_{2}, \cdots, \mu_{n}\right\}$ where the $\mu_{k}$ 's are distinct. Hence, in (*) we may replace $X$ by a suitable $X_{1}$ and meanwhile assume that $\lambda_{k}$ is real for $k=1,2, \cdots, n$. Then, by (1.3) we may replace $X_{1}$ by another suitable $X_{2}$ and replace $\sum_{k=1}^{n} \lambda_{k} E\left(\delta_{k}\right)$ and $\sum_{k=1}^{n} \lambda_{k} F\left(\delta_{k}\right)$ by their Cayley transforms. By (1.4) these Cayley transforms are power bounded by $K$. Hence, our first assertion follows from (1.2) with $\sin \theta \geqq 1 / K^{2}$. If $T$ and $S$ are normal operators the constant $K$ in (1.2) and (1.4) may be taken to be 1 . It follows that $\theta=\pi / 2$.

CoRollary 1.6. If $N$ is a normal operator in $\mathscr{B}(\mathscr{H})$ and $\lambda_{1}$ and $\lambda_{2}$ are distinct eigenvalues of $\Delta_{N}$ with corresponding eigenspaces $\mathscr{X}_{1}$ and $\mathscr{X}_{2}$, then $\mathscr{X}_{1}$ is orthogonal to $\mathscr{X}_{2}$ and $\mathscr{X}_{2}$ is orthogonal to $\mathscr{Z}_{1}$.

Proof. $\mathscr{Z}_{1}=\mathscr{N}\left(\Delta_{N}-\lambda_{1} I\right)-\mathscr{N}\left(C(N, N)-\lambda_{1} I\right)=\mathscr{N}\left(C\left(N-\lambda_{1} I, N\right)\right)$ ( $I$ denotes either the identity on $\mathscr{H}$ or the identity on $\mathscr{B}(\mathscr{H})$ ). If $\Delta_{N}(X)=\lambda_{2} X$ then $C\left(N-\lambda_{1} I, N\right)(X)=\left(\lambda_{2}-\lambda_{1}\right) X$ so $X$ is in the range of $C\left(N-\lambda_{1} I, N\right)$. Hence, by (1.5) $\mathscr{X}_{2}$ is orthogonal to $\mathscr{X}_{1}$. Similarly, $\mathscr{X}_{1}$ is orthogonal to $\mathscr{Z}_{2}$.

2. If $S$ and $T$ are scalar operators on $\mathscr{X}$, then [3, p. 112] $C(T, S)$ is a generalized scalar operator and, hence, a decomposable operator. Thus, $\mathscr{B}_{C}(\{\lambda\})$ is a spectral maximal subspace for each $\lambda$ in the spectrum of $C(T, S)$. In this section we prove that $\mathscr{B}_{C}(\{\lambda\})$ consists solely of eigenvectors (i.e. $(D)$ holds for $C(T, S)$ ) and give two examples.

THEOREM 2.1. $\mathscr{B}_{C}(\{\lambda\})=\mathscr{N}(C-\lambda I)$ for each $\lambda$ in $\sigma(C(T, S))$, if $T$ and $S$ are scalar operators in $\mathscr{B}(\mathscr{X})$.

Proof. Since $T-\lambda I$ is a scalar operator if $T$ is a scalar operator we may assume $\lambda=0$. Let $E(\cdot)$ and $F(\cdot)$ be the spectral resolutions of the identity associated with $T$ and $S$ respectively. Suppose that $X \in \mathscr{B}(\mathscr{Z})$ and that $\left\|C^{n}(T, S)(X)\right\|^{1 / n} \rightarrow 0$ as $n \rightarrow \infty$. Then [3, 4.5, p. 113] $E(\delta) X F(\delta)=X F(\delta)$ for each closed subset $\delta$ of $C$. Hence,

$$
E(\delta) X F(\gamma)=E(\delta \cap \gamma) X F(\gamma)=0
$$

for all Borel subsets $\gamma$ with closure disjoint from $\delta$. Let $\delta$ be a closed subset of $C$ and let $\left\{\gamma_{k}\right\}$ be disjoint Borel sets which cover the complement of $\delta$ and have closures from $\delta$. Then 


$$
\begin{aligned}
E(\delta) X & =E(\delta) X F\left(U \gamma_{k}\right)+E(\delta) X F(\delta) \\
& =E(\delta) X\left(\sum_{k=1}^{\infty} F\left(\gamma_{k}\right)\right)+E(\delta) X F(\delta) \\
& =E(\delta) X F(\delta) .
\end{aligned}
$$

Therefore, $E(\delta) X=X F(\delta)$. Since Borel measures are regular and $E(\cdot)$ and $F(\cdot)$ are countably additive, $E(\delta) X=X F(\delta)$ for each Borel set $\delta \subset C$ and so $T X=X S$, i.e., $C(T, S)(X)=0$. The theorem now follows from $[3,4.4$, p. 113].

EXAMPLES Let $\tau=\{\lambda \in C:|\lambda|=1\}$ and let $U$ be multiplication by $\lambda$ on $L^{2}(\tau)$. Thus, $U$ is the simple bilateral shift. Let $E(\cdot)$ be the spectral measure associated with $U$. We show that $\Delta_{U}$ has no non-zero eigenvalues. For if $\Delta_{U}(X)=-\lambda X$ with $\lambda \neq 0$ then as in (2.1) $E(\delta) X=X E(\delta+\lambda)$ for each Borel set $\delta(\delta+\lambda$ is the translate of $\delta$ by $\lambda$ ). Therefore,

$$
X=E(\tau) X E(\tau)=X E((\tau+\lambda) \cap \tau) .
$$

But $(\tau+\lambda) \cap \tau$ consists of at most two points and $E(\{\mu\})=0$ for each $\mu$ in $C$; thus $X=0$.

On the other hand, if $M$ is multiplication by $x$ on $L^{2}(0,1)$ and $W_{a}$ is the operator defined on $L^{2}(0,1)$ by

$$
\left(W_{a} f\right)(x)=\int_{a}^{1} f(x-t) d \mu_{a}(t)=\left\{\begin{array}{cl}
f(x-a) & \text { if } a \leqq x \leqq 1 \\
0 & \text { if } x<a
\end{array}\right.
$$

where $a \in(0,1)$ is fixed, then a simple computation shows

$$
M W_{a}-W_{a} M=a W_{a} .
$$

Thus, depending (in part) on the geometry of the spectrum of $T, \mathscr{B}_{\Delta_{T}}(\{\lambda\})$ may or may not be empty.

3. Our goal in this section is to show that $(E)$ holds for $C(T, S)$.

THEOREM 3.1. If $T$ is a decomposable operator in $\mathscr{B}(\mathscr{Z})$ such that the range of $T$ is closed and the range of $T$ meets the null space of $T$ at angle $\theta>0$, then 0 is an isolated point of the spectrum of $T$.

Proof. Since $\mathscr{R}(T)$ is closed, by the open mapping theorem there is a constant $M>0$ such that each $y$ in $\mathscr{R}(T)$ has the form $y=T x$ where $x \in \mathscr{Z}$ and $\|x\| \leqq M\|y\|$. Also, since $\mathscr{R}(T)$ meets $\mathscr{N}(T)$ at a positive angle, $\mathscr{N}(T)$ meets $\mathscr{R}(T)$ at angle $\varphi>0$. 
For each $r>0$ let $F_{r}=\{\lambda \in C:|\lambda| \geqq r\}$ and let $T_{r}=\left.T\right|_{\chi_{T}\left(F_{r}\right)}$. Then $\sigma\left(T_{r}\right) \subseteq F_{r}$ so $T_{r}^{-1}$ exists and is bounded. Let $1 / r_{0}=M / \sin \varphi$. We show that $\left\|T_{r}^{-1}\right\| \leqq 1 / r_{0}$ for all $r>0$. If $y \in \mathscr{Z}_{T}\left(F_{r}\right)$ then $y \in \mathscr{R}(T)$ so $y=T x$ for some $x \in \mathscr{P}$ with $\|x\| \leqq M\|y\|$. Let $w=x-T_{r}^{-1} y$. Then $T w=0$ so, since $\mathscr{N}(T)$ meets $\mathscr{R}(T)$ at angle $\varphi$

$$
\sin \varphi\left\|T_{r}^{-1} y\right\| \leqq\left\|w+T_{r}^{-1} y\right\|=\|x\| \leqq M\|y\| \text {. }
$$

Since $y$ was arbitrary, $\left\|T_{r}^{-1}\right\| \leqq M / \sin \varphi=1 / r_{0}$. It follows that $\sigma\left(T_{r}\right) \subset F_{r_{0}}$ for all $r>0$. Indeed, if $T_{r}-\lambda I$ were not invertible for some $\lambda$ in $C$ with $0<|\lambda|<r_{0}$ then because $T_{r}$ is a decomposable operator, $\left\|\left(T_{r}-\lambda I\right) x_{n}\right\| \rightarrow 0$ for a sequence $x_{n}$ of unit vectors in $\mathscr{Z}_{T}\left(F_{r}\right)$ and

$$
\begin{aligned}
|1 /| \lambda\left|-\left\|T_{r}^{-1} x_{n}\right\|\right| & \leqq(1 /|\lambda|)\left\|x_{n}-\lambda T_{r}^{-1} x_{n}\right\| \\
& \leqq\left(\| T_{r}^{-1}|| /|\lambda|\right)\left\|\left(T_{r}-\lambda I\right) x_{n}\right\|
\end{aligned}
$$

so that $\| T_{r}^{-1}|| \geqq 1 /|\lambda|>1 / r_{0}$, a contradiction. Thus, $\sigma(T) \subset F_{r_{0}} U$ $\{\lambda \in C:|\lambda|<r\}$ for all $r>0$ and so 0 is an isolated point of $\sigma(T)$.

EXAMPLE 3.2. Let $V$ be the Volterra operator defined on $L^{2}(0,1)$ by

$$
(V f)(x)=\int_{0}^{x} f(t) d t
$$

Then $V$ is an injective compact quasinilpotent operator with dense range. Hence, $V$ is a decomposable operator such that 0 is an isolated point of its spectrum and $\mathscr{R}(V)$ is orthogonal to $\mathscr{N}(V)(=\{0\})$. However, since $V$ is a compact operator, its range cannot be closed.

Recall that Lumer and Rosenblum have shown [6] that for any $T$ and $S$ in $\mathscr{B}(\mathscr{Q})$

$$
\sigma(C(T, S))=\sigma(T)-\sigma(S)=\{\lambda-\mu: \lambda \in \sigma(T), \mu \in \sigma(S)\} .
$$

It follows easily that 0 is an isolated point of $\sigma(C(T, S))$ if and only if $\sigma(T) \cap \sigma(S)$ consists of points which are isolated in both $\sigma(T)$ and $\sigma(S)$. When this occurs we shall say that $T$ and $S$ have almost disjoint spectra.

THEOREM 3.3. If $T$ and $S$ are scalar operators in $\mathscr{B}(\mathscr{P})$, then $C(T, S)$ has closed range if and only if 0 is an isolated point of $\sigma(C(T, S))$. In particular $C(T, T)$ has closed range if and only if $\sigma(T)$ is finite.

Proof. As remarked previously, $C(T, S)$ is a generalized scalar 
operator; hence a decomposable operator. Furthermore, by (1.5) its range meets its null space at an angle $\theta>0$. Thus, if $\mathscr{R}(C(T, S))$ is closed 0 is an isolated point of $\sigma(C(T, S))$ by (3.1). Conversely, suppose that 0 is an isolated point of $\sigma(C(T, S))$. Then $T$ and $S$ have almost disjoint spectra. Let $\left\{\lambda_{1}, \cdots, \lambda_{n}\right\}=\sigma(T) \cap \sigma(S)$ and let $E(\cdot)$ and $F(\cdot)$ be the spectral resolutions of the identity associated with $T$ and $S$ respectively. Put $P_{k}=E\left(\left\{\lambda_{k}\right\}\right), Q_{k}=F\left(\left\{\lambda_{k}\right\}\right)$ for $1 \leqq$ $k \leqq n, P_{0}=I-\sum_{k=1}^{n} P_{k}$, and $Q_{0}=I-\sum_{k=1}^{n} Q_{k}$. Let $\mathscr{B}_{i j}=P_{i} \mathscr{B}(\mathscr{Z}) Q_{j}$ for $0 \leqq i \leqq n$ and $0 \leqq j \leqq n$. Then each $\mathscr{B}_{i j}$ is an invariant subspace for $C(T, S)$ and the span of the $\mathscr{B}_{i j}$ 's is $\mathscr{B}(\mathscr{Z})$. Hence, it suffices to show that $C_{i j}=\left.C(T, S)\right|_{\mathscr{F}_{i j}}$ has closed range for $0 \leqq i$, $j \leqq n$. Now if $i \neq 0, j \neq 0$ and $Y \in \mathscr{B}_{i j}$ then $C_{i j}(Y)=\left(\lambda_{i}-\lambda_{j}\right) Y$. Thus, in these cases $C_{i j}$ has closed range. Also, if $1 \leqq i \leqq n$ and $Y \in \mathscr{B}_{j 0}, C_{i 0}(Y)=\lambda_{i} P_{i} Y-Y S=\lambda_{i} P_{i} Y Q_{0}-P_{i} Y Q_{0} S=P_{i} Y Q_{0}\left(\lambda_{i} I-S\right)$. Since $\left.\left(\lambda_{i} I-S\right)\right|_{Q_{0} \mathscr{E}}$ is invertible $\left(\lambda_{i}\right.$ is isolated in $\left.\sigma(S)\right) C_{i 0}$ has closed range. Similarly, $C_{0 j}$ has closed range for $1 \leqq j \leqq n$. To complete the proof we show that $C_{00}$ has closed range. Note that $\sigma\left(\left.T\right|_{P_{0} \mathscr{x}}\right) \cap$ $\sigma\left(\left.S\right|_{Q_{0} \not}\right)=\varnothing$. Choose a real number $k$ so that $k>\|S\|+\|T\|$ and define $S_{1}=S P_{0}+k\left(I-P_{0}\right)$ and $T_{1}=Q_{0} T-k\left(I-Q_{0}\right)$. Then $\sigma\left(S_{1}\right) \cap \sigma\left(T_{1}\right)=\varnothing$ and $C\left(T_{1}, S_{1}\right)$ is invertible on $\mathscr{B}(\mathscr{Q})$. Thus, for each $X$ in $\mathscr{B}(\mathscr{Q})$ there is $Y$ in $\mathscr{B}(\mathscr{Q})$ so that $T_{1} Y-Y S_{1}=X$. Hence, $P_{0} X Q_{0}=T P_{0} Y Q_{0}-P_{0} Y Q_{0} S$ and so $C_{00}$ is onto $\mathscr{B}_{00}$.

4. In this section we restrict ourselves to scalar operators $T$ and $S$ acting on a complex infinite dimensional Hilbert space $\mathscr{H}$. Recall that an operator $T$ in $\mathscr{B}(\mathscr{H})$ is of scalar type if and only if $T$ is similar to a normal operator [10].

TheOREM 4.1. Suppose that $N_{1}$ and $N_{2}$ are normal operators on a complex, infinite dimensional Hilbert space $\mathscr{H}$ and that $\sigma\left(N_{1}\right) \cap \sigma\left(N_{2}\right)$ contains a point $\lambda$ which is an accumulation point for at least one of these spectra and is either an accumulation point or else an isolated eigenvalue of infinite multiplicity of the other. Then there is an operator $V$ in $\mathscr{B}(\mathscr{H})$ such that the span of the null space and the range of $C\left(N_{1}, N_{2}\right)$ is orthogonal to the span of $V$. In particular $\mathscr{R}\left(C\left(N_{1}, N_{2}\right)\right)+\mathscr{N}\left(C\left(N_{1}, N_{2}\right)\right)$ is not dense in $\mathscr{B}(\mathscr{H})$.

Proof. We assume that $\lambda=\lim \lambda_{n}$ where $\left\{\lambda_{n}\right\}$ is a a sequence of distinct elements in $\sigma\left(N_{1}\right)$. The proof for the other case is similar. If $\lambda$ is an accumulation point of $\sigma\left(N_{2}\right)$ choose a sequence $\left\{\mu_{n}\right\}$ of distinct elements in $\sigma\left(N_{2}\right)$ so that $\lambda=\lim \mu_{n}$. Taking subsequences if necessary, we may assume that the $\mu_{n}$ 's are also distinct from the $\lambda_{n}$ 's. If $\lambda$ is an isolated eigenvalue of infinite multiplicity for $N_{2}$ take $\lambda=\mu_{n}$. 
Let $E_{i}(\cdot)$ be the spectral measure associated with $N_{i}$ for $i=1,2$. Choose disks $D_{n}$ of radius $r_{n}$ about $\lambda_{n}$ so that $D_{n} \cap D_{m}=\varnothing$ if $n \neq m$ and $\mu_{n} \notin D_{m}$ for any $n$ or $m$. Note that since $\lambda_{n} \rightarrow \lambda, r_{n} \rightarrow 0$. For each $n$ put $P_{n}=E_{1}\left(D_{n}\right)$, and choose a unit vector $f_{n}$ in the range of $P_{n}\left(P_{n} \neq 0\right.$ since $\left.\lambda_{n} \in \sigma\left(N_{1}\right)\right)$. If $\lambda$ is an accumulation point of $\sigma\left(N_{2}\right)$ choose disks $D_{n}^{\prime}$ of radius $r_{n}^{\prime}$ about $\mu_{n}$ so that $D_{n}^{\prime} \cap D_{m}^{\prime}=\varnothing$ and $D_{n}^{\prime} \cap D_{n}=\varnothing$ for all $m$ and $n$. Put $Q_{n}=E_{2}\left(D_{n}^{\prime}\right)$. If $\lambda$ is an isolated eigenvalue of infinite multiplicity for $N_{2}$ let $\left\{Q_{n}\right\}$ be an infinite set of nonzero, mutually orthogonal projections such that $Q_{n} \leqq E_{2}(\{\lambda\})$ for each $n$. In either case for each $n$ choose a unit vector $e_{n}$ in the range of $Q_{n}$. Define $V$ on $\mathscr{H}$ by $V e_{n}=f_{n}$ and $V x=0$ for $x$ in the orthogonal complement of the span of $\left\{Q_{n} \mathscr{H}\right\}_{n=1}^{\infty}$. Then $V \in \mathscr{B}(\mathscr{H})$ and $\|V\|=\left\|P_{n} V Q_{n}\right\|=1$ for all $n$. In fact $V$ is a partial isometry. Let $X$ and $W$ be in $\mathscr{B}(\mathscr{H})$ and suppose that $N_{1} W=W N_{2}$. Then (as in (2.1)) $E_{1}(\delta) W=W E_{2}(\delta)$ for each Borel set $\delta \subset C$. Let

$$
\alpha=\left\|V-W-\left(N_{1} X-X N_{2}\right)\right\| \text {. }
$$

To complete the proof we show that $\alpha \geqq 1$.

$$
\begin{aligned}
\alpha & =\alpha\left\|P_{n}\right\|\left\|Q_{n}\right\| \geqq\left\|P_{n} V Q_{n}-P_{n} W Q_{n}-\left(P_{n} N_{1} X Q_{n}-P_{n} X N_{2} Q_{n}\right)\right\| \\
& \geqq 1-\left\|W E_{2}\left(D_{n}\right) Q_{n}\right\|-\left\|N_{1} P_{n} X Q_{n}-P_{n} X Q_{n} N_{2}\right\| .
\end{aligned}
$$

Now $E_{2}\left(D_{n}\right) Q_{n}=0$ since $D_{n} \cap D_{n}^{\prime}=\varnothing$ and $\lambda \notin D_{n}$ so

$$
\begin{aligned}
\alpha \geqq 1 & -\left\|N_{1} P_{n} X Q_{n}-\lambda_{n} P_{n} X Q_{n}\right\|-\left\|\left(\lambda_{n}-\mu_{n}\right) P_{n} X Q_{n}\right\| \\
& -\left\|\mu_{n} P_{n} X Q_{n}-P_{n} X Q_{n} N_{2}\right\| .
\end{aligned}
$$

Thus,

$$
\begin{aligned}
1-\alpha & \leqq\left(\left\|N_{1} P_{n}-\lambda_{n} P_{n}\right\|+\left|\lambda_{n}-\mu_{n}\right|+\left\|\mu_{n} Q_{n}-N_{2} Q_{n}\right\|\right)\left\|P_{n} X Q_{n}\right\| \\
& \leqq\left(r_{n}+\left|\lambda_{n}-\mu_{n}\right|+r_{n}^{\prime}\right)\left\|P_{n} X_{n} Q_{n}\right\|
\end{aligned}
$$

which goes to 0 as $n \rightarrow \infty$. Hence, $\alpha \geqq 1$.

THEOREM 4.2. Let $T$ and $S$ be scalar operators in $\mathscr{B}(\mathscr{H})$. The following are equivalent.

(a) $C((T-\lambda I), S)$ has closed range for each $\lambda \in C$

(b) $C(T, S)$ is a spectral operator

(c) $C(T, S)$ is a scalar operator

(d) $\sigma(T) \cup \sigma(S)$ is finite.

Proof. Clearly, $T-\lambda I$ and $S$ have almost disjoint spectra for all $\lambda$ in $C$ if and only if $\sigma(T) \cup \sigma(S)$ is finite so (a) is equivalent to (d) by (3.3). If $\sigma(T) \cup \sigma(S)$ is finite then so is $\sigma(C(T, S))$. In this 
case the Riesz-Dunford theory implies that $C(T, S)$ is a spectral operator and, in virtue of (2.1) it is even scalar. Hence, (d) implies (b). Since the implication from (c) to (b) is trivial, to complete the proof we need only show that (b) implies (d). Hence, assume that $C(T, S)$ is a spectral operator. Since $T$ and $S$ are scalar operators in $\mathscr{B}(\mathscr{H})$, there are normal operators $N_{i}$ and invertible operators $X_{i}$ in $\mathscr{B}(\mathscr{H})$ for $i=1,2$ so that

$$
T=X_{1} N_{1} X_{1}^{-1} \text { and } S=X_{2} N_{2} X_{2}^{-1} \text {. }
$$

Define $\Theta$ acting on $\mathscr{B}(\mathscr{H})$ by $\Theta(Y)=X_{1}^{-1} Y X_{2}$. Then

$$
\Theta C(T, S) \Theta^{-1}=C\left(N_{1}, N_{2}\right)
$$

and so $C\left(N_{1}, N_{2}\right)$ is a spectral operator. Let $E(\cdot)$ be the spectral resolution of the identity associated with $C\left(N_{1}, N_{2}\right)$. Then [3, p. 33]

$$
B_{C}(\delta)=E(\delta) \mathscr{B}(\mathscr{H})
$$

for all closed subsets $\delta$ in $C$. Hence, by (2.1)

$$
\mathscr{N}\left(C\left(N_{1}, N_{2}\right)\right)=\mathscr{B}_{C}(\{0\})=E(\{0\}) \mathscr{B}(\mathscr{H}) .
$$

On the other hand, $\mathscr{B}_{C}(\delta) \subset \mathscr{R}\left(C\left(N_{1}, N_{2}\right)\right)$ for each closed subset $\delta$ of $C$ with $0 \notin \delta$ so that the countable additivity of $E(\cdot)$ implies

$$
E(\{C \backslash\{0\}\}) \mathscr{B}(\mathscr{C}) \subset \mathscr{R}(C)^{-}
$$

where the bar denotes the closure in the uniform topology. Therefore, the algebraic direct sum

$$
\mathscr{R}(C)^{-}+\mathscr{N}(C)=\mathscr{B}(\mathscr{C}) \text {. }
$$

Now $C_{\lambda}=C\left(\left(N_{1}-\lambda I\right), N_{2}\right)=C\left(N_{1}, N_{2}\right)-\lambda I$ is a spectral operator for each $\lambda$ in $C$ so as before

$$
\mathscr{R}\left(C_{\lambda}\right)^{-}+\mathscr{N}\left(C_{\lambda}\right)=\mathscr{B}(\mathscr{C})
$$

for each complex $\lambda$. If both $\sigma\left(N_{1}\right)$ and $\sigma\left(N_{2}\right)$ were infinite then for some $\lambda, \sigma\left(N_{1}-\lambda I\right) \cap \sigma\left(N_{2}\right)$ would contain a common accumulation point and by $(4.1)(*)$ would be false. Thus, either $N_{1}$ or $N_{2}$ has finite spectrum. Say $\sigma\left(N_{2}\right)$ is finite. Then, since $\mathscr{H}$ is infinite dimensional $N_{2}$ has an isolated eigenvalue of infinite multiplicity. If $\sigma\left(N_{1}\right)$ were infinite (4.1) would again contradict (*). Thus, $\sigma(T) \cup \sigma(S)=\sigma\left(N_{1}\right) \cup \sigma\left(N_{2}\right)$ is finite.

Remarks 4.3. Clearly, (a) and (d) remain equivalent if $T$ and $S$ are scalar operators acting on a Banach space. We do not know, however, if (b) and (c) are also equivalent to (d) in this more 
general setting. Unfortunately, our proof does not appear to generalize. Our proof depends on the existence of an operator $V$ which is not in the closed linear span of $\mathscr{R}(C)$ and $\mathscr{N}(C)$. Although $V$ can be formally defined in the Banach space setting, it is not clear that $V$ will be bounded.

5. In this section we investigate the extent to which properties (F) and (G) hold for $C(T, S)$ and use our results to give an example of a Hermitian operator whose square is not Hermitian. We begin by recalling the definition of the numerical range of an element in a Banach algebra.

DEFINITION 5.1. Let $\mathfrak{A}$ be a complex Banach algebra with identity I. The set of states on $\mathfrak{A}$ is by definition

$$
\mathscr{P}^{\circ}=\left\{f \in \mathfrak{U}^{*}: f(I)=1=\|f\|\right\} .
$$

The numerical range of an element $a$ in $\mathfrak{N}$ is by definition the set

$$
W_{0}(a)=\{f(a): f \in \mathscr{P}\} \text {. }
$$

Since $\mathscr{P}$ is a weak* closed convex subset of the unit ball of $\mathfrak{A}^{*}$, $W_{0}(a)$ is a closed convex subset of $C$ for each $a \in \mathfrak{A}$. If $\mathfrak{A}=\mathscr{B}(\mathscr{H})$ $W_{0}(\cdot)$ is just the closure of $W(\cdot)$ the usual numerical range (for further information see [9]). The numerical radius of $a$ is by definition $\sup \left\{|\lambda|: \lambda \in W_{0}(a)\right\}$. The spectral radius of $a$ is by definition $\sup \{|\lambda|: \lambda \in \sigma(a)\}$. An element $a$ in $\mathfrak{N}$ is Hermitian if $W_{0}(a)$ is real. Recall [7] that $a$ in $\mathfrak{A}$ is Hermitian if and only if $\|\exp (i t a)\|=1$ for all $t$ in $\boldsymbol{R}$. For an operator $T$ in $\mathscr{B}(\mathscr{Z})$ define the operators $L_{T}$ and $R_{T}$ in $\mathscr{B}(\mathscr{B}(\mathscr{X}))$ by $L_{T}(X)=T X$ and $R_{T}(X)=X T$.

Theorem 5.2. If $T$ is in $\mathscr{B}(\mathscr{X})$ then $W_{0}(T)=W_{0}\left(L_{T}\right)=W_{0}\left(R_{T}\right)$.

Proof. For each state $f$ on $\mathscr{B}(\mathscr{B}(\mathscr{Q})$ ) the formula $g(X)=$ $f\left(L_{X}\right)$ determines a state $g$ on $\mathscr{B}(\mathscr{X})$. Hence, $W_{0}\left(L_{T}\right) \subset W_{0}(T)$. Conversely for each state $f$ on $\mathscr{B}(\mathscr{Z})$ the formula $g\left(L_{X}\right)=f(X)$ determines a state $g$ on $\left\{L_{X}: X \in \mathscr{B}(\mathscr{X})\right\}$ which then extends by the Hahn-Banach theorem to a state on all of $\mathscr{B}(\mathscr{B}(\mathscr{Q}))$. It follows that $W_{0}(T) \subset W_{0}\left(L_{T}\right)$ and so $W_{0}(T)=W_{0}\left(L_{T}\right)$. Similarly, $W_{0}(T)=W_{0}\left(R_{T}\right)$.

COROLLARY 5.3. If $T$ and $S$ are Hermitian operator in $\mathscr{B}(\mathscr{Z})$, then $C(T, S)$ is a Hermitian operator.

Proof. $C(T, S)=L_{T}-R_{S}$. Thus 


$$
W_{0}(C(T, S)) \subset W_{0}\left(L_{T}\right)-W_{0}\left(R_{S}\right)=W_{0}(T)-W_{0}(S) \subset R .
$$

THEOREM 5.4. If $N_{1}$ and $N_{2}$ are normal operators in $\mathscr{B}(\mathscr{H})$ where $\mathscr{H}$ is a complex Hilbert space then $C\left(N_{1}, N_{2}\right)=H+i J$ where $H$ and $J$ are commuting Hermitian operators.

Proof. Write $N_{j}=A_{j}+i B_{j}$, where $A_{j}$ and $B_{j}$ are the real and imaginary parts of $N_{j}$ for $j=1,2$. Let $H=C\left(A_{1}, A_{2}\right)$ and $J=$ $C\left(B_{1}, B_{2}\right)$. Then $H$ and $J$ are Hermitian operators by (5.3) and since $A_{j} B_{j}=B_{j} A_{j}$ for $j=1,2, H$ and $J$ commute.

THEOREM 5.5. If $N_{1}$ and $N_{2}$ are normal operators on a complex Hilbert space then the spectral radius and the numerical radius of $C\left(N_{1}, N_{2}\right)$ are equal.

Proof. Palmer has shown [7, Lemma 1.6] that the conclusion of the theorem holds for all operators of the form $H+i J$ when $H$ and $J$ are commuting Hermitian operators. Hence, the theorem follows from (5.4).

EXAMPLE 5.6. The norm and the spectral radius of $C\left(N_{1}, N_{2}\right)$ need not be equal, however. Indeed, Stampfli has shown [8] that

$$
\left\|C\left(N_{1}, N_{2}\right)\right\|=\inf _{\lambda \in C}\left\{\left\|N_{1}-\lambda I\right\|+\left\|N_{2}-\lambda I\right\|\right\} .
$$

Thus, if $N$ is a normal operator in $\mathscr{B}(\mathscr{H})$ whose spectrum is an equilateral triangle of side 1 then (Lumer and Rosenblum) the spectral radius of $\Delta_{N}=C(N, N)$ is 1 but since the norm of $N-\lambda I$ is equal to the spectral radius of $N-\lambda I,\left\|\Delta_{N}\right\|=2 / \sqrt{3}$. Note that in this case $\sigma\left(\Delta_{N}\right)$ is a solid hexagon centered at the origin. On the other hand, $C\left(N_{1}, N_{2}\right)$ is a convexoid operator.

THeOREM 5.7. If $N_{1}$ and $N_{2}$ are normal ope Hilbert space then the convex hull of $\sigma(C(\Lambda$ on a complex $W_{0}\left(C\left(N_{1}, N_{2}\right)\right)$.

Proof. Let conv (.) denote the convex hull of the set within the parentheses. Since $\sigma(a) \subset W_{0}(a)$ for any element $a$ of a Banach algebra $\mathfrak{A}\left[9\right.$, Theorem 1] it suffices to show $W_{0}\left(C\left(N_{1}, N_{2}\right)\right) \subset$ conv $\left(\sigma\left(C\left(N_{1}, N_{2}\right)\right)\right)$. Now

$$
\begin{aligned}
W_{0}\left(C\left(N_{1}, N_{2}\right)\right) & \subset W_{0}\left(N_{1}\right)-W_{0}\left(N_{2}\right)=W\left(N_{1}\right)^{-}-W\left(N_{2}\right)^{-} \\
& =\operatorname{conv}\left(\sigma\left(N_{1}\right)\right)-\operatorname{conv}\left(\sigma\left(N_{2}\right)\right) \\
& =\operatorname{conv}\left(\sigma\left(N_{1}\right)-\sigma\left(N_{2}\right)\right)=\operatorname{conv}\left(\sigma\left(C\left(N_{1}, N_{2}\right)\right)\right) .
\end{aligned}
$$


where we have used (5.2) and property (G) of the introduction.

THeOREM 5.8. If $P$ is a (self-adjoint) projection on a complex Hilbert space $\mathscr{H}$ then $L_{P} R_{P}$ is a Hermitian operator if and only if $P$ is 0 or $I$.

Proof. We give the proof in the case that $\mathscr{H}$ has dimension 2 and $P \mathscr{C}$ has dimension 1 . The generalization to higher dimensions is obvious. As remarked in (5.1) it suffices to show that $\left\|\exp \left(i t L_{P} R_{P}\right)\right\|>\mathbf{1}$ for some $t$ in $\boldsymbol{R}$. Let

$$
T=\left[\begin{array}{ll}
(1 / 2) i & 1 / 2 \\
1 / 2 & 1 / 2
\end{array}\right]
$$

on $P \mathscr{H} \oplus(I-P) \mathscr{H}$ and let $t=3 \pi / 2$. Then since $\exp \left(i t L_{P} R_{P}\right)=$ $I+\left(e^{i t}-1\right) L_{P} R_{P}$

$$
\exp \left(\text { it } L_{P} R_{P}\right)(T)=\left[\begin{array}{ll}
1 / 2 & 1 / 2 \\
1 / 2 & 1 / 2
\end{array}\right]
$$

which is a projection and, hence, has norm 1 . On the other hand, $\left\|T^{*} T\right\|^{2}<3 / 4$ as an easy computation shows. Hence, $\| \exp \left(\right.$ it $\left.L_{P} R_{P}\right) \|>1$ for $t=3 \pi / 2$ and $L_{P} R_{P}$ is not Hermitian.

EXAMPle 5.9. Let $P$ be a projection as in (5.8). Then $\Delta_{P}$ is a Hermitian operator by (5.3) but $\Delta_{P}^{2}$ is not a Hermitian oprator. Indeed, since the Hermitian operators on $\mathscr{B}(\mathscr{H})$ form a real vector space and

$$
L_{P} R_{P}=\frac{1}{2}\left(\Delta_{P}-\Delta_{P}^{2}\right)
$$

it follows from (5.8) that $\Delta_{P}^{2}$ is not Hermitian.

In conclusion we remark that Crabbe [4] and Browder [2] have given similar examples.

\section{REFERENCES}

1. Joel Anderson, On normal derivations, Proc. Amer. Math. Soc., 38 (1973), 135-140.

2. Andrew Browder, States, numerical range, etc., Proceedings of the Brown informal Analysis Seminar, Summer, 1969.

3. I. Colojoařa and C. Foiaș, Theory of Generalized Spectral Operators, Gordon and Breach, New York, 1968.

4. M. J. Crabbe, Some results on the numerical range of an operator, J. London Math. Soc., (2) 2 (1970), 741-745.

5. Nelson Dunford, Spectral operators, Pacific J. Math., 4 (1954), 321-354.

6. G. Lumer and M. Rosenblum, Linear operator equations, Proc. Amer. Math. Soc., 10 (1959), 32-41. 
7. T. W. Palmer, Unbounded normal operators on Banach space, Trans. Amer. Math. Soc., 133 (1968), 385-414.

8. J. G. Stampfli, The norm of a derivation, Pacific J. Math., 24 (1970), 209-214.

9. J. G. Stampfli and J. P. Williams, Growth conditions and the numerical range in a Banach algebra, Tôhoku Math. J., 20 (1968), 417-424.

10. John Wermer, Commuting spectral measures on Hilbert space, Pacific J. Math., 4 (1954), 355-361.

Received October 25, 1974.

California Institute of Technology

AND

INSTITUTE OF MATHEMATICS

BUCHAREST, RUMANIA 



\section{PACIFIC JOURNAL OF MATHEMATICS}

\section{EDITORS}

RICHARD ARENS (Managing Editor)

University of California

Los Angeles, California 90024

\author{
R. A. Beaumont \\ University of Washington \\ Seattle, Washington 98105
}

\section{J. DugundjI}

Department of Mathematics University of Southern California Los Angeles, California 90007

D. Gilbarg and J. Milgram

Stanford University

Stanford, California 94305

\section{ASSOCIATE EDITORS}
E. F. BECKENBACH
B. H. NeumanN
F. WOLF
K. YoshidA

\section{SUPPORTING INSTITUTIONS}

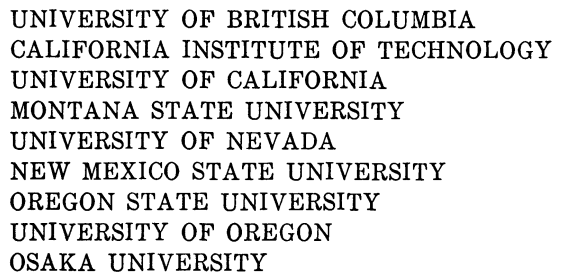

UNIVERSITY OF BRITISH COLUMBIA CALIFORNIA INSTITUTE OF TECHNOLOGY UNIVERSITY OF CALIFORNIA MONTANA STATE UNIVERSITY UNIVERSITY OF NEVADA NEW MEXICO STATE UNIVERSITY OSAKA UNIVERSITY OREGON STATE UNIVERSITY UNIVERSITY OF OREGON

\author{
UNIVERSITY OF SOUTHERN CALIFORNIA \\ STANFORD UNIVERSITY \\ UNIVERSITY OF TOKYO \\ UNIVERSITY OF UTAH \\ WASHINGTON STATE UNIVERSITY \\ UNIVERSITY OF WASHINGTON \\ AMERICAN MATHEMATICAL SOCIETY
}

The Supporting Institutions listed above contribute to the cost of publication of this Journal, but they are not owners or publishers and have no responsibility for its content or policies.

Mathematical papers intended for publication in the Pacific Journal of Mathematics should be in typed form or offset-reproduced, (not dittoed), double spaced with large margins. Please do not use built up fractions in the text of your manuscript. You may however, use them in the displayed equations. Underline Greek letters in red, German in green, and script in blue. The first paragraph or two must be capable of being used separately as a synopsis of the entire paper. Items of the bibliography should not be cited there unless absolutely necessary, in which case they must be identified by author and Journal, rather than by item number. Manuscripts, in triplicate, may be sent to any one of the editors. Please classify according to the scheme of Math. Reviews, Index to Vol. 39. All other communications should be addressed to the managing editor, or Elaine Barth, University of California, Los Angeles, California, 90024.

The Pacific Journal of Mathematics expects the author's institution to pay page charges, and reserves the right to delay publication for nonpayment of charges in case of financial emergency.

100 reprints are provided free for each article, only if page charges have been substantially paid. Additional copies may be obtained at cost in multiples of 50 .

The Pacific Journal of Mathematics is issued monthly as of January 1966. Regular subscription rate: $\$ 72.00$ a year $(6$ Vols., 12 issues). Special rate: $\$ 36.00$ a year to individual members of supporting institutions.

Subscriptions, orders for back numbers, and changes of address should be sent to Pacific Journal of Mathematics, 103 Highland Boulevard, Berkeley, California, 94708.

PUBLISHED BY PACIFIC JOURNAL OF MATHEMATICS, A NON-PROFIT CORPORATION

Printed at Kokusai Bunken Insatsusha (International Academic Printing Co., Ltd.), 8-8, 3-chome, Takadanobaba, Shinjuku-ku, Tokyo 160, Japan. 


\section{Pacific Journal of Mathematics}

\section{Vol. 61, No. 2 December, 1975}

Graham Donald Allen, Francis Joseph Narcowich and James Patrick Williams, An operator version of a theorem of Kolmogorov .......................

Joel Hilary Anderson and Ciprian Foias, Properties which normal operators share with normal derivations and related operators . . . . . . . . . . . . . . . . . . . . .

Constantin Gelu Apostol and Norberto Salinas, Nilpotent approximations and

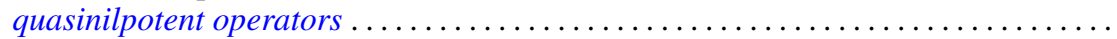

James M. Briggs, Jr., Finitely generated ideals in regular $F$-algebras . . . . . . . . . . .

Frank Benjamin Cannonito and Ronald Wallace Gatterdam, The word problem and power problem in 1-relator groups are primitive recursive ..................

Clifton Earle Corzatt, Permutation polynomials over the rational numbers ...........

L. S. Dube, An inversion of the $S_{2}$ transform for generalized functions . . . . . . . . . . William Richard Emerson, Averaging strongly subadditive set functions in unimodular

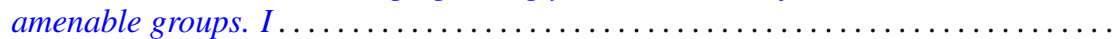

Barry J. Gardner, Semi-simple radical classes of algebras and attainability of

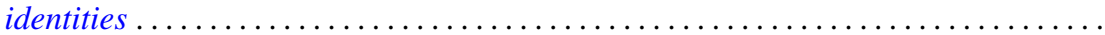

Irving Leonard Glicksberg, Removable discontinuities of A-holomorphic functions ....

Fred Halpern, Transfer theorems for topological structures . . . . . . . . . . . . . . .

H. B. Hamilton, T. E. Nordahl and Takayuki Tamura, Commutative cancellative

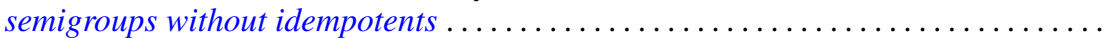

Melvin Hochster, An obstruction to lifting cyclic modules .....................

Alistair H. Lachlan, Theories with a finite number of models in an uncountable power

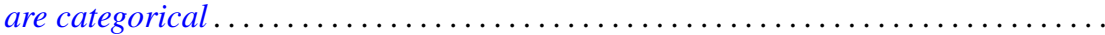

Kjeld Laursen, Continuity of linear maps from $C^{*}$-algebras . . . . . . . . . . . . .

Tsai Sheng Liu, Oscillation of even order differential equations with deviating arguments ....

Jorge Martinez, Doubling chains, singular elements and hyper- $Z$

Mehdi Radjabalipour and Heydar Radjavi, On the geometry of num Thomas I. Seidman, The solution of singular equations, I. Linear equations in Hilbert

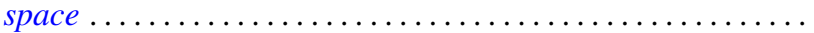

R. James Tomkins, Properties of martingale-like sequences ......

Alfons Van Daele, A Radon Nikodým theorem for weights on von Neumann

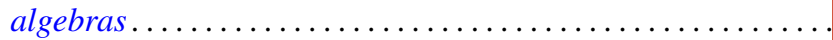

Kenneth S. Williams, On Euler's criterion for quintic nonresidues

Scott Andrew Wolpert, Noncompleteness of the Weil-Petersson metric for Teichmüller

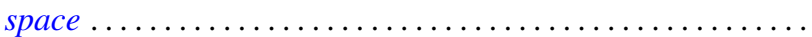

Volker Wrobel, Some generalizations of Schauder's theorem in locally convex

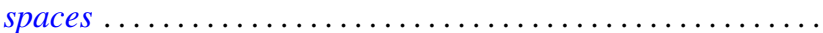

Kelly Denis McKennon, Corrections to: "Multipliers of type $(p, p)$ "; "Multipliers of type $(p, p)$ and multipliers of the group $L_{p}$-algebras"; "Multipliers and the

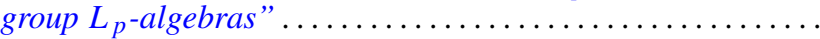

Andrew M. W. Glass, W. Charles (Wilbur) Holland Jr. and Stephen H. McCleary, Correction to: " $a *$-closures to completely distributive lattice-ordered

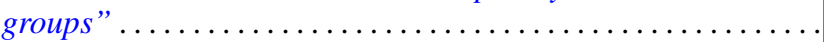

Zvi Arad and George Isaac Glauberman, Correction to: "A characteristic subgroup of

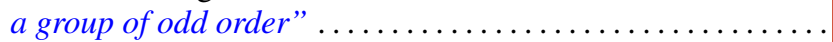

Roger W. Barnard and John Lawson Lewis, Correction to: "Subordination theorems

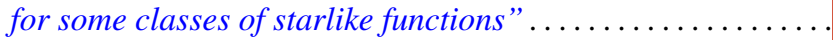

